\title{
Optimization Design and Development of Sensing Coil and Analog Signal Conditioning Electronics for Fluxgate Magnetometer Sensor
}

\section{Sadiq UA ${ }^{1,2,3}$, Sadka $\mathrm{AH}^{4}$ and Oluyombo OW ${ }^{1 *}$}

${ }^{1}$ National Space Research \& Development Agency, Abuja, Nigeria

${ }^{2}$ Center for Satellite Technology Development, Abuja, Nigeria

${ }^{3}$ Electrical \& Electronic Engineering Department, University of Abuja, Nigeria

${ }^{4}$ Department of Electronic \& Computer Engineering, Brunel University London, United Kingdom

\begin{abstract}
The design of fluxgate magnetometers is typically a nonlinear multi-objective optimization problem. Different objectives often conflict with each other, and sometimes an optimal Fluxgate Magnetometer Sensor (FMS) performance is difficult to achieve. The sensitivity of the sensor decreases with an increase of noise level while trying to reduce the sensor dimension. Hence, there is need for a systematic optimization approach for FMS design to find its optimum performance. The combined modified multi-objective Firefly Optimization Algorithm (FOA) and systematic optimization approach is suggested to improve FMS's design in this research by simultaneously optimizing the sensitivity and noise of a FMS while the sensor core, pick-up coil, and detection circuit are minimized. The developed model allowed improved sensitivity of $86.65 \%$, reduction of noise level by $59.97 \%$ while still keeping the sensor size small by $14.29 \%$.
\end{abstract}

Keywords: Fluxgate magnetometer sensor; Noise; Sensitivity; Firefly optimization algorithm

\section{Introduction}

FMS are commonly used magnetic field sensors for measuring DC or low frequency magnetic field vectors [1]. FMS have very high sensitivity spans a wide range from $100 \mathrm{pT}$ to $100 \mu \mathrm{T}$ [2], low noise, small size, small power requirements, and high temperature stability [3]. Moreover, the advancements in magnetic materials which form the heart of FMS and characterize their sensitivity, noise level, and linearity range responsible for their popularity among other competitive magnetic field sensors [4]. These make them one of the magnetic field sensors that still attract the attention of many researchers because of their wide applications in space research and navigation systems [5], particularly in Earth's magnetic field exploration surveys [6,7].

In order to optimize the performance of magnetometers, different optimization techniques for their structures and core materials had been developed. For example, the conventional approach was based on Part-by-Part Optimization (PPO) technique, which includes designing the sensor core first, then select the dimension of pick-up coil, and finally develop a low noise detection circuit. However, PPO technique is too slow, time consuming, and expensive [8]. Another optimization technique for the magnetometer parameters proposed by Chen et al., [9] and Grosz et al., [10] was based on an analytical model, which was numerically solved to obtain improved large set of parameters such as volume and weight of pick-up coil, power consumption, and the noise of the signal conditioning circuit. However, the analytical optimization technique becomes unnecessarily complex when performing large number of numerical calculations to optimize the magnetometer, hence, introducing difficulty in interpreting the results obtained [8]. Recently, optimization of Fluxgate Magnetometer Sensors (FMS) had been based on Finite Element Method (FEM) by using simulation software such as ANSYS, FEMM, Flux 2D, and others. On the other hand, most of these tools could not offer the users the ability to fully express their optimization purposes by formulating the objective functions [6].

Driving the excitation coil and detecting the pick-up coil signal require careful design of the excitation and detection electronics circuits $[11,12]$. In most literatures, the excitation circuits for fluxgate sensors are typically based on a sinusoidal [13], triangular [11] or pulsed excitation [14]. The pulsed excitation is easier to generate than sinusoidal or triangular excitation [15] and represents a trade-off between the sensitivity and power consumption [16]. A pulsed excitation reduces power consumption at the expense of low sensitivity $[11,16]$.

The block diagram of the entire fluxgate magnetometer is shown in Figure 1 . To periodically saturate the ferromagnetic material, the excitation signal is fed into the excitation coil of the fluxgate sensor with a square excitation current waveform with excitation frequency f. The pick-up coil of the FMS detects the signal by the rising and falling edges of core magnetizing current see Figure $2 c$. The pick-up coil is fed into a pre-amp with mild tuning to the second harmonic (2f) of the excitation frequency because the second harmonic fluxgate magnetometers produce the highest sensitivity and the lowest noise

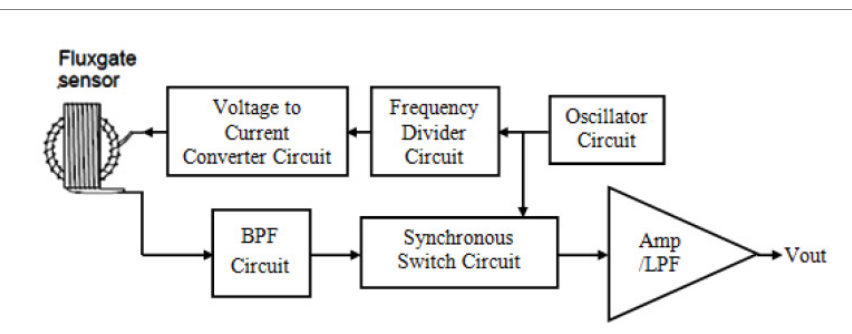

Figure 1: Typical Second Harmonic Demodulator Scheme.

*Corresponding author: Oluyombo OW, National Space Research \& Development Agency, Abuja, Nigeria, Tel: 09-2342220; E-mail: oluyombowaheed@gmail.com

Received November 15, 2018; Accepted December 21, 2018; Published December 29, 2018

Citation: Sadiq UA, Sadka AH, Oluyombo OW (2018) Optimization Design and Development of Sensing Coil and Analog Signal Conditioning Electronics for Fluxgate Magnetometer Sensor. J Biosens Bioelectron 9: 261. doi: 10.4172/21556210.1000261

Copyright: ( 2018 Sadiq UA, et al. This is an open-access article distributed under the terms of the Creative Commons Attribution License, which permits unrestricted use, distribution, and reproduction in any medium, provided the original author and source are credited. 
[1]. Therefore, it is possible to extract the information on the external magnetic field by a synchronous demodulation [17]. Demodulation is usually accomplished with a phase sensitive detector, typically, a CMOS analogue switch following the pre-amp [18].

In this paper, the design of fluxgate magnetometers is assumed to be a nonlinear multi-objective optimization problem. Different objectives often conflict with each other, and sometimes optimal magnetometer performance is not achieved. Metaheuristic algorithms are very powerful in dealing with non-linear multi-objective optimization problem [19]. The multi-objective Firefly Optimization Algorithm (FOA) was proposed in this research, because FOA is one of the nature-inspired metaheuristic algorithms, which is capable of handling the design problems in electromagnetics with a large number of design variables and multiple objectives under complex nonlinear constraints [19]. For instance, sensitivity and noise of a fluxgate magnetometer can be improved while the sensor core, pick-up coil, and detection circuit are minimized. The combined multi-objective FOA and systematic optimization approach is suggested to improve FMS's design in this work by simultaneously finding the dimensions and geometry of the sensor core, pick-up coil, and detection circuit in order to reduce its noise and increase its sensitivity.

\section{System Design}

\section{Square wave current generator}

Frequency generator circuit was designed using different electronics components such as operational amplifier, transistors, hex inverters, and so on. The IC 4069 is a CMOS logic chip having six independent inverters [20]. It was used for interfacing and to make simple square wave generators as shown in Figure 2.

The frequency determined by $\mathrm{R}_{1}$ and $\mathrm{C}_{1}$ is:

$$
F=\frac{1}{1.39 \times R_{1} \times C_{1}}
$$

Where $\mathrm{F}$ is the frequency in $\mathrm{Hz}, \mathrm{R}_{1}$ is the timing resistor in ohms and $\mathrm{C}_{1}$ is the timing capacitor in Farad.

As shown in Figure 2, the circuit used a few components such as two resistors with $R_{1}$ used as timing resistor and a capacitor $C_{1}$ and consumes less power. The output frequency produced by the square wave oscillator depends on two components $R_{1}$ and $C_{1}$. Changing the values of the resistor $\mathrm{R}_{1}$ and capacitor $\mathrm{C}_{1}$ will result in change of output frequency.

\section{Voltage to current converter}

The frequency divider output produce analog output voltage but the current was weak and cannot be directly used to drive the magnetic core to saturation. The oscillator and the frequency divider can only produce a few tens of milli-amperes at most, while the sensor core requires many amperes. Hence, there is need for current amplification by using power transistors. Therefore, a complementary emitterfollower is commonly used for efficient bipolar current amplification. Figure 3 shows a low-noise class-AB power amplifier using NPN and PNP transistors $\mathrm{Q}_{1}$ and $\mathrm{Q}_{2}$ respectively. The two transistors $\mathrm{Q}_{1}$ and $\mathrm{Q}_{2}$ were configured as a complementary emitter-follower. Class $A B$ amplifier is very similar to class B amplifiers, but their performance is improved by the addition of two diodes that eliminate the crossover region and allows both transistors to be turned on at the same time. The efficiency (around 50\%) is not as high as class B because both

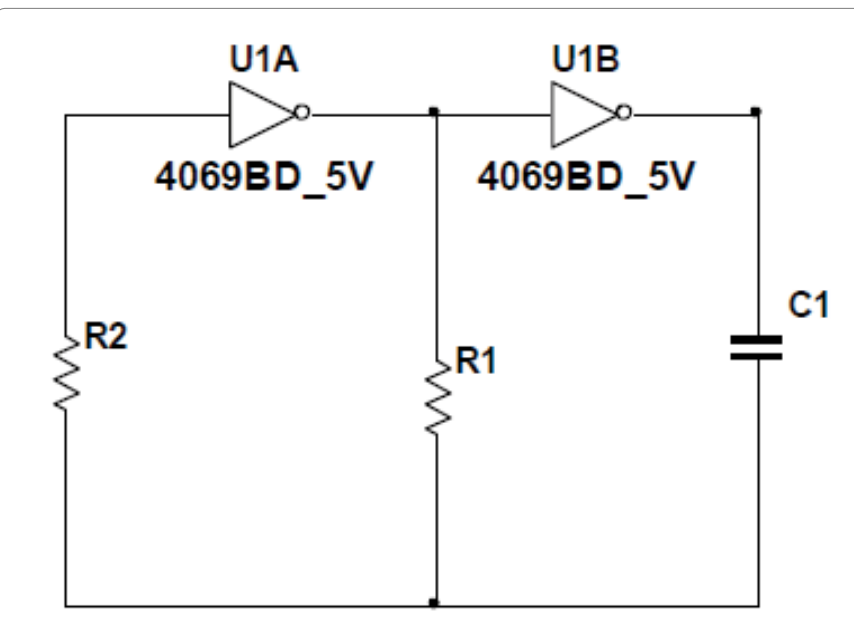

Figure 2: Schematic diagram of a square wave generator.

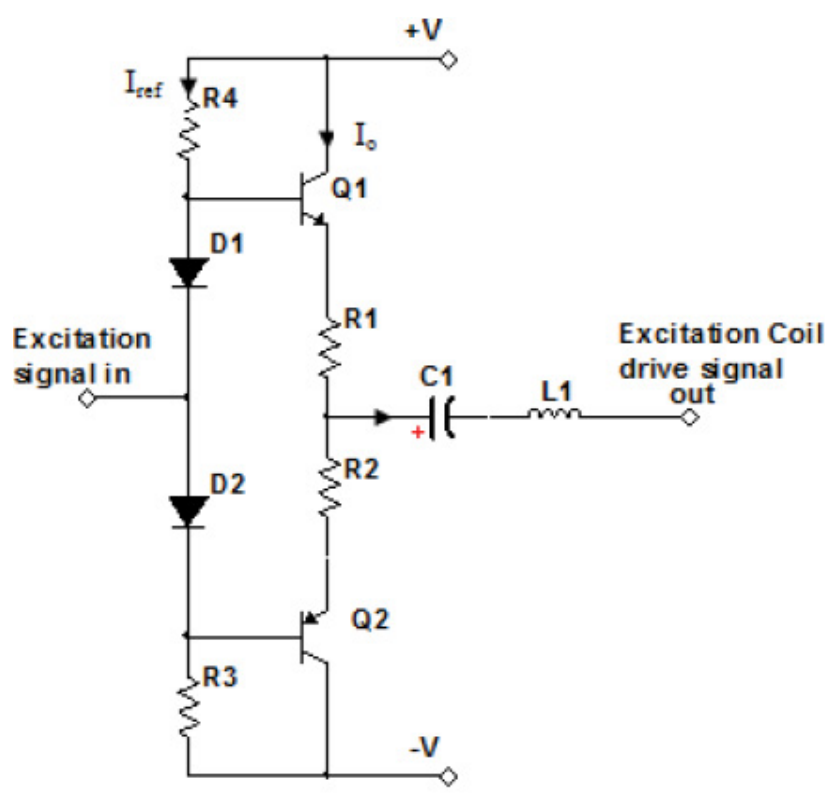

Figure 3: Schematic diagram of a voltage to current converter.

transistors are turned on simultaneously, but accuracy is improved. It is the most commonly used voltage to current converter amplifier.

As shown in Figure 3, resistor $\mathrm{R}_{4}$ and diode $\mathrm{D}_{1}$ biased the NPN transistor $\mathrm{Q}_{1}$ while $\mathrm{D}_{2}$ and $\mathrm{R}_{3}$ biased the transistor $\mathrm{Q}_{2}$. The values of the biasing resistors $\mathrm{R}_{3}$ and $\mathrm{R}_{4}$ are calculated as:

$$
R_{4}=\frac{V_{c c}-V_{b e}}{I_{r e f}}
$$

Where $R_{4}$ is a bias resistor and $R_{3}$ is equal to $\mathrm{R}_{4} . \mathrm{V}_{c c}$ is the supplied voltage, $\mathrm{V}_{\mathrm{be}}$ is the emitter-base voltage and Iref is the transistor base bias current.

Resistors $\mathrm{R}_{1}$ and $\mathrm{R}_{2}$ are equal in values and set the operating current for the output of the transistors. The values of the emitter biasing resistors $\mathrm{R}_{1}$ and $\mathrm{R}_{2}$ are calculated as: 


$$
R_{1}=\frac{V}{I_{o}} \ln \left(\frac{I_{r e f}}{I_{o}}\right)
$$

Where $R_{1}$ is an emitter bias resistor and $R_{1}$ is equal to $R_{2}$. V is the supplied voltage, Io is the output current, and $\mathrm{I}_{\text {ref }}$ is the transistor base bias current.

The two diodes serve to bias the transistors and reduce the crossover distortion that occurs when the input waveform crosses zero. Without the diodes, the oscillator output would have to swing $1.4 \mathrm{~V}$ to turn one transistor on and then bring the other transistor off.

Connecting the excitation circuit to the excitation coil requires coupling capacitor that serves to isolate the AC signal from any DC bias voltages. In order for a coupling capacitor to operate effectively, it must have the right size. Capacitor $\mathrm{C}_{1}$ served to block the $\mathrm{DC}$ component of the current source from reaching the excitation resonant circuit. The equation to calculate the value of the coupling capacitor $\mathrm{C}_{1}$ is:

$$
C_{1}=\frac{1}{3.2 f_{\text {exc }} R_{\text {coil }}}
$$

Where $\mathrm{C}$ is the capacitance in Farads, and $\mathrm{f}_{\text {exc }}$ is the excitation signal frequency in Hertz. $\mathrm{R}_{\text {coil }}$ is the impedance on the load side of the capacitor which in this case is the excitation coil resistance.

As shown in Figure 3, when transistor $\mathrm{Q}_{1}$ is turned on, capacitor $\mathrm{C}_{3}$ is charged smoothly as the charging current is limited by inductor $\mathrm{L}_{1}$. The main function of the inductor $\mathrm{L}_{1}$ is to limit the current drawn from the source for the fluxgate excitation current. This was achieved by the use of a high impedance (larger than the fluxgate sensor) inductor, which operates in the non-saturated mode over part of the excitation period. In the non-saturated state, the high impedance of the inductor limits the current flowing from the source to the excitation circuit.

\section{Detection electronics}

The pick-up coil of the fluxgate sensor detects the signal induced by the flux collapse (saturation) and flux recovery (de-saturation) of the core magnetizing current. This small induced voltage output signal of the pick-up coil was detected and it was compensated by amplifying and filtering the signal. The second harmonic component of the induced voltage across the pick-up coil was conditioned by using the electronics circuit shown in Figure 4.

The output voltage from the pick-up coil is usually small compared to the reference voltage of the Analog to Digital Converter (ADC). A low noise operational amplifier is needed to amplify the output voltage of the pick-up coil. As shown in Figure 4, at very low frequencies (within the cut-off frequency or frequency response of the amplifier), the capacitor $\mathrm{C}_{2}$ is an open circuit and the gain of the signal conditioning circuit was high, which made it acted as an amplifier. In order to evaluate the performance of FMS associated with the detection circuit, the frequency response of FMS detection amplifier was computed as [9,21]:

$$
V_{o}=\frac{R_{2}}{R_{1}} \cdot \frac{V_{\text {pick-up coil }}}{1+\left(\frac{L_{w}+R_{2} C_{2}}{R_{1}}\right) j \omega-\frac{R_{2}}{\left(R_{1}\right)} \cdot L_{w} C_{2} \omega^{2}}
$$

Where, $R_{z}=R_{w}+R_{g}, R_{w}$ is the pick-up coil winding resistance, $L_{w}$ is the pick-up coil inductance, $C_{f}$ is the amplifier feedback capacitor, $R_{f}$ is the amplifier feedback resistor.

\section{Power supply}

To provide a stable voltage to the excitation circuit, a voltage regulator circuit was used to maintain a stable supply voltage to excitation circuit. The power supply was used to provide all the voltages necessary for driving the various components along with reference voltages. This adds up to the regulated supply voltages of $+5 \mathrm{~V}, \pm 10 \mathrm{~V}$, and ground.

\section{Experimental Setup}

Component values were optimized to improve performance and to minimize currents in the fluxgate magnetometer. Then the circuit was tested on a breadboard and soldered on a prototyping board. After an iterative process of design optimization, the excitation frequency selected was $5 \mathrm{kHz}$ square wave. The schematic diagram of the complete excitation circuit is shown in Figure 5.

As shown in Figure 5, in order to ensure proper saturation of the ferrite magnetic core material the whole circuit was driven by a square wave oscillator at $20 \mathrm{kHz}$ frequency. A duty cycle of $50 \%$ on both the $5 \mathrm{kHz}$ and the $10 \mathrm{kHz}$ output waveforms was ensured. A duty cycle different from $50 \%$ could compromise the demodulation of the signals produced by the sensing coils and, hence, it has to be avoided. As shown in Figure 5, by using equation (1), the oscillator block was built around resistors R1, R2 and potentiometer VR1 with capacitor C1 and Integrated Circuit (IC), hex-inverter 4069BD (U1A \& U1B). The oscillator circuit was tuned to twice the excitation frequency $\left(2 \mathrm{f}_{\text {exc }}\right)$ and variable between $1 \mathrm{kHz}$ and $20 \mathrm{kHz}$ by means of a $100 \mathrm{k} \Omega$ potentiometer (VR1).

The completed ring cores with excitation coils wound

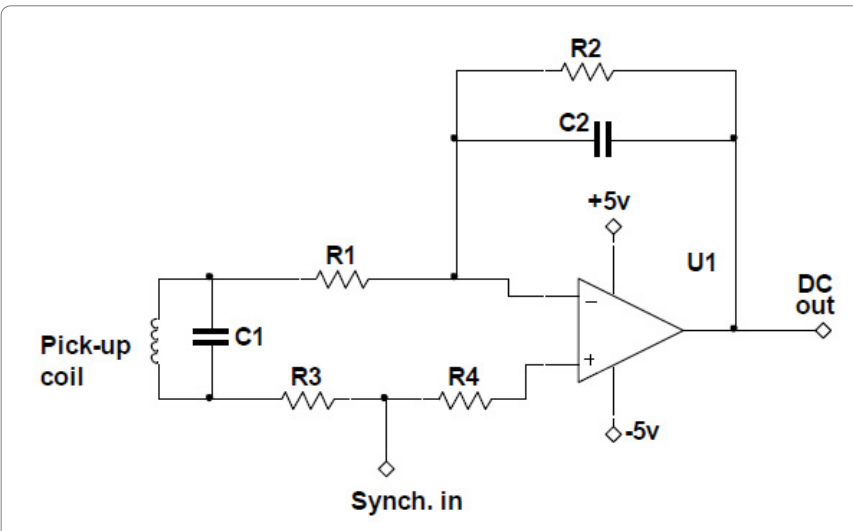

Figure 4: Schematic diagram of detection electronic.

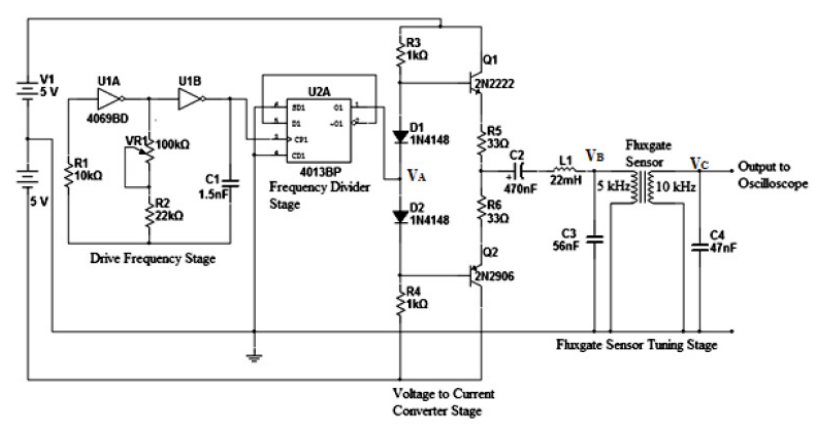

Figure 5: Driver circuit of developed fluxgate sensors. 
Citation: Sadiq UA, Sadka AH, Oluyombo OW (2018) Optimization Design and Development of Sensing Coil and Analog Signal Conditioning Electronics for Fluxgate Magnetometer Sensor. J Biosens Bioelectron 9: 261. doi: 10.4172/2155-6210.1000261

circumferentially were put inside a pick-up coil bobbin, which were constructed to hold the pick-up coil as shown in Figure 6. Finally, the pick-up coil with 646 turns was wound diametrically on the core with copper wire having $0.2 \mathrm{~mm}$.

As shown in Figure 6, the final dimension of the fluxgate sensor was $0.023 \mathrm{~m} \times 0.02 \mathrm{~m} \times 0.01 \mathrm{~m}$, the dimension of the fluxgate sensor together with the printed circuit board was $0.085 \mathrm{~m} \times 0.063 \mathrm{~m} \times 0.01 \mathrm{~m}$, while the whole system package was $0.093 \mathrm{~m} \times 0.071 \mathrm{~m} \times 0.022 \mathrm{~m}$. The completed fluxgate sensor prototype is shown in Figure 7.

The block diagram of the fluxgate magnetometer electronic testing board is shown in Figure 8. It consists of a $5 \mathrm{kHz}$ driving oscillator, frequency divider, current booster, fluxgate sensor, synchronization circuit, and detection amplifier circuit.

The actual experimental setup is shown in Plate 9 with Tektronix (0-72V , 1.2A) Programmable DC Power Supply (model: PSW 4721) used to power the sensor through the testing board with the calculated excitation current of $99 \mathrm{~mA}$ for the sensors (Figure 9).

The excitation and pickup coil signal waveforms were examined by connecting the Tektronix Four Channel Digital Signal Oscilloscope (model: TPS 2024B) to the sensor excitation stage (at points a, b, and c shown in Figure 8) on the testing board. The tuned second harmonic output voltage responses of the developed FOA fluxgate sensors as a function of the applied external magnetic field were verified. As seen in Figure 9, Tektronix Digital Multimeter (2050 model) (with root mean square (rms) range selected) was connected to the pick-up coil output terminal (point ' $c$ ' shown in Figure 8) of the prototype sensors to measure the peak output voltages of the sensors corresponding to the external magnetic field supplied from the Helmholtz coil. Magnetic field strength meter (calibrated magnetometer) was used to evaluate

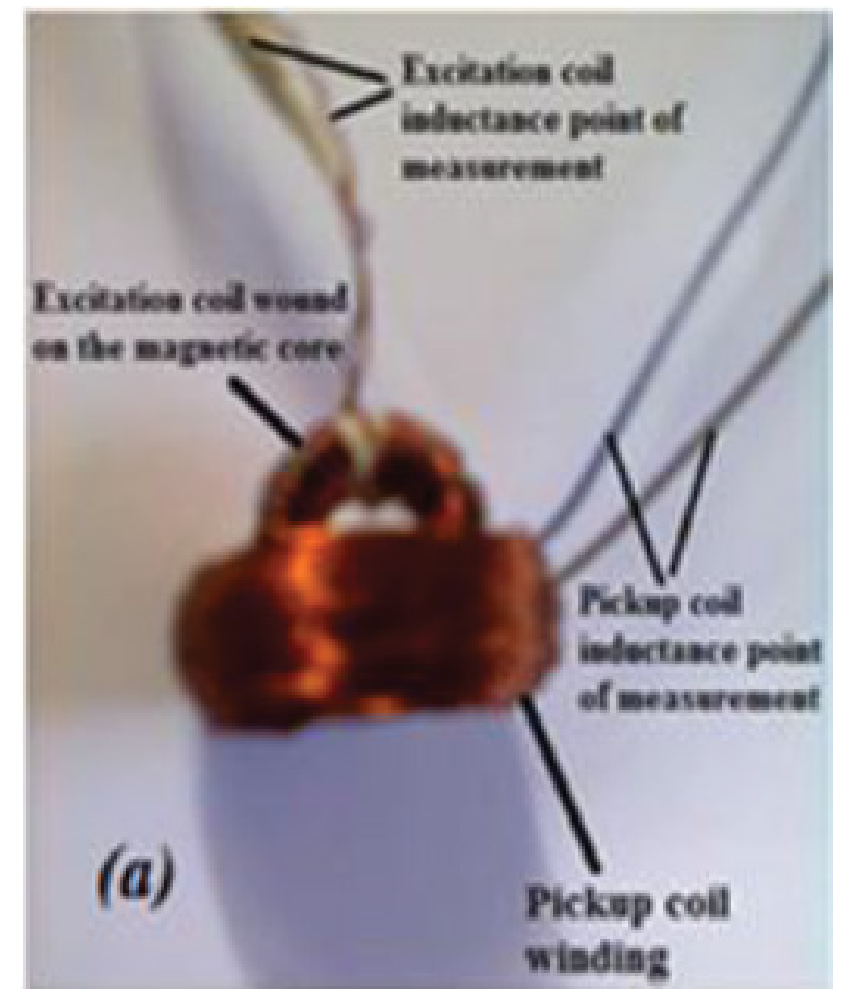

Figure 6: Prototype sensor coil assembly.

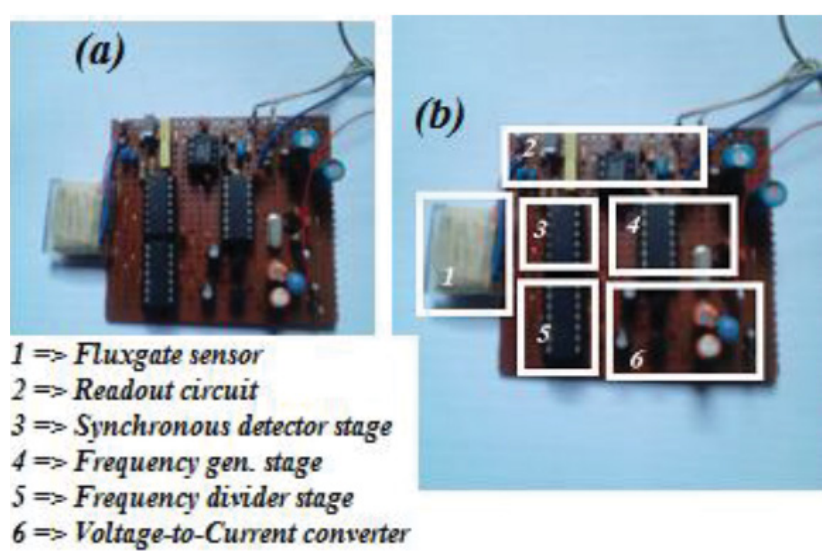

Figure 7: Complete fluxgate sensor system.

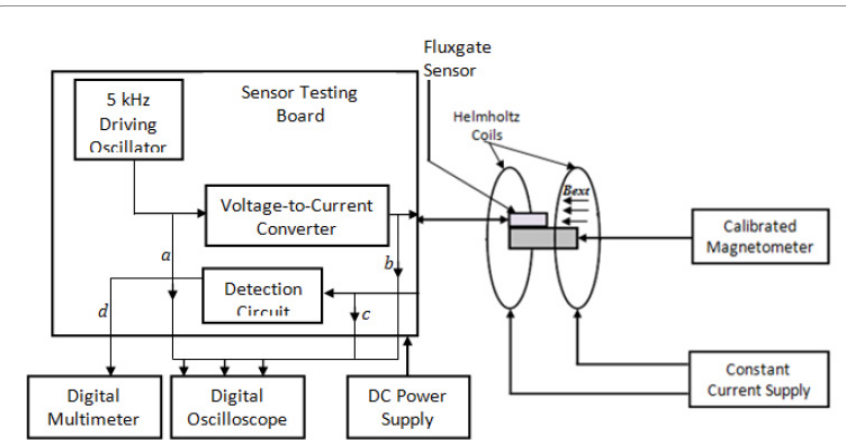

Figure 8: Block Diagram of the Experimental Setup for the Sensor Characterization.

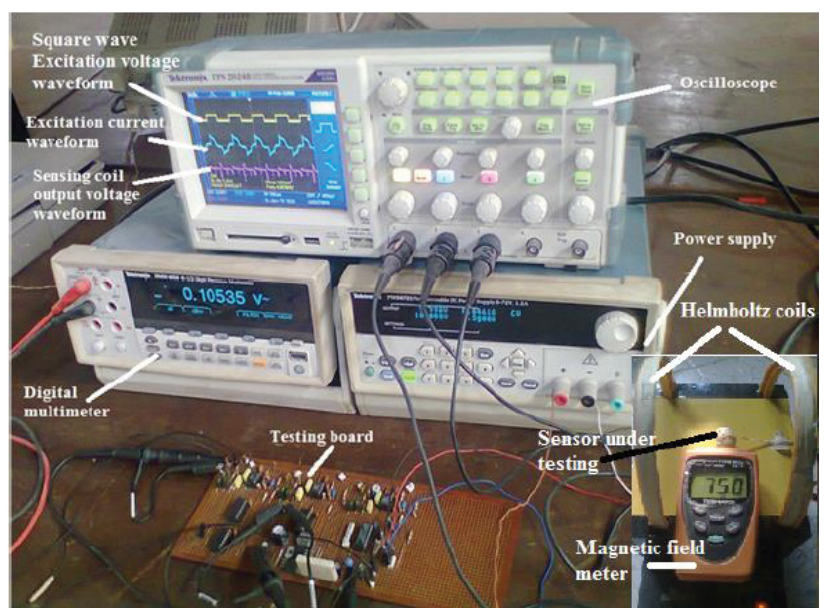

Figure 9: Actual Experimental Setup for the Characterization of the Fluxgate Sensors.

the magnitude of the magnetic field supplied from the Helmholtz coil to the fluxgate sensors. This was done by placing the magnetic field strength meter (sensor) perpendicular to the magnetic field of the Helmholtz coil.

\section{Results}

Based on the optimum values of the dimensions and geometric 
parameters obtained from the FOA design, FMS prototype was fabricated and studied. The sensor was made from MnZn ferrite with a thickness of $2 \mathrm{~mm}$. The FOA was written in Matlab environment and the results were obtained by running the developed program on a $1.50 \mathrm{GHz}$ Intel $^{\circledR}$ core $^{\text {nt }}$ Duo CPU Windows 7 Ultimate 32-bit personal computer. The sensors were designed to withstand up to $99 \mathrm{~mA}$ current. Copper wire having $0.411 \mathrm{~mm}$ was wound as the excitation coil on each sensor with 49 turns. Parameters and the values of the FOA model are contained in Table 1.

The measurements of the output response to field variations were made with $15 \mu \mathrm{T}$ as the minimum magnetic field up to $75 \mu \mathrm{T}$ maximum with step of $10 \mu \mathrm{T}$. The $15 \mu \mathrm{T}$ resolution of these measurements was limited by output capability of the Helmholtz coils at the minimum driving current. It was noticed that the minimum driving current for the Helmholtz coils was $8 \mathrm{~mA}$. Below this minimum driving current ( $8 \mathrm{~mA})$, the Helmholtz coils operation became unstable resulting in the fluctuations of the output magnetic field of the calibrated magnetometer used. In addition, the $75 \mu \mathrm{T}$ maximum magnetic fields of these measurements were limited by the induced excitation field of $\pm 67.99 \mu \mathrm{T}$ inside the sensor core. This was validated by the principle of fluxgate magnetometer. The smaller the sensitivity the higher the magnetic field ranges of sensor. It was noticed that the magnetic field linearity of these sensors was increasing as the core dimension was decreasing. This was due to the increasing nature of the excitation current as the core dimension decreases which in turn increases the linearity of the sensor. Table 2 presents the tuned second harmonic output voltage obtained from the experimental measurements (Figure 9) of the pick-up coil output voltage.

This data shows that the FOA simulation routine provided an accurate geometric dimension of the sensor core and the pick-up coil. During the experimental data measurements, it was observed that the sensitivity increased as the core dimension decreased up to an optimum dimension of the core at which the voltage sensitivity began to decrease.

The plot shown on Figure 10 obtained from Table 2 shows the response of the pick-up coils of the developed sensors when tuned to second harmonic of the excitation frequency under the imposed external magnetic field from Helmholtz coils.

\begin{tabular}{|c|c|c|}
\hline Parameters & FOA Sensor & Unit \\
\hline Core outside diameter & 12.00 & $\mathrm{~mm}$ \\
\hline Core inside diameter & 8.00 & $\mathrm{~mm}$ \\
\hline Core height & 2.00 & $\mathrm{~mm}$ \\
\hline Number of Pick-up coil turns & 646 & - \\
\hline Pick-up Coil bobbin thickness & 5.00 & $\mathrm{~mm}$ \\
\hline Amplifier Feedback resistor & 100.00 & $\mathrm{k} \Omega$ \\
\hline Amplifier feedback capacitor & 150.0 & $\mathrm{nF}$ \\
\hline Amplifier input resistor & 1.80 & $\mathrm{k} \Omega$ \\
\hline
\end{tabular}

Table 1: Optimum values of FOA designed sensor parameters.

\begin{tabular}{|c|c|}
\hline External Magnetic Field $(\boldsymbol{\mu} \mathbf{T})$ & Output Voltages $(\mathbf{m V})$ \\
\hline 15 & 18.10 \\
\hline 25 & 29.32 \\
\hline 35 & 38.89 \\
\hline 45 & 51.34 \\
\hline 55 & 63.23 \\
\hline 65 & 74.33 \\
\hline 75 & 86.45 \\
\hline
\end{tabular}

Table 2: Pick-up coil output voltages obtained from measurements.

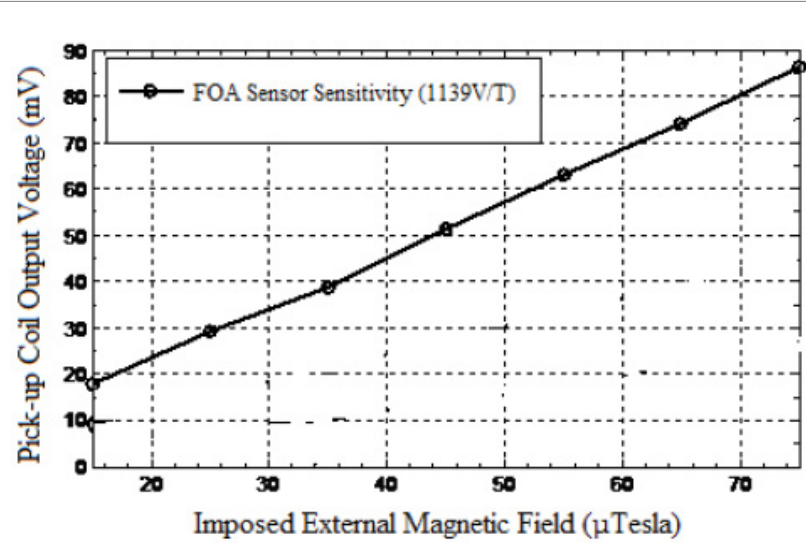

Figure 10: Fluxgate Parallel Tuned Output Plotted against External Magnetic Field.
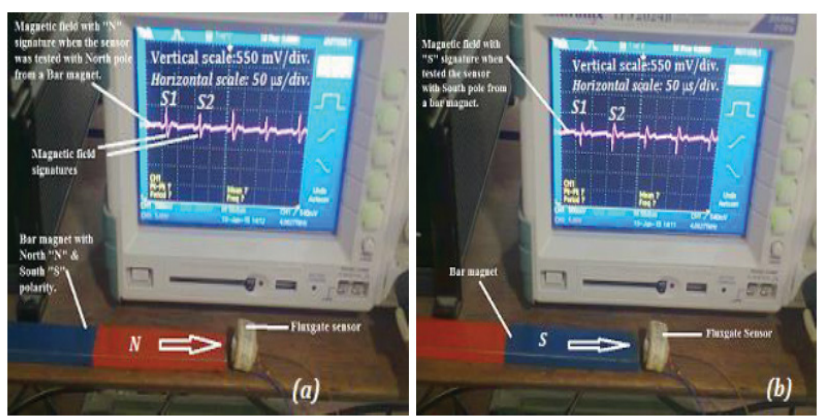

Figure 11: Magnetic Field Sensing from a Bar Magnet.

The FMS exhibited voltage sensitivity of $1139 \mathrm{~V} / \mathrm{T}$ at $75 \mu \mathrm{T}$ external magnetic field range and $5 \mathrm{kHz}$ excitation frequency.

Figure 11 shows the FMS responses to external magnetic field due to a bar magnet (scaled to $50 \mu$ s per division on the horizontal axis and $550 \mathrm{mV}$ per division on the vertical axis). These responses reflected both the influences from the applied field of a bar magnet and the remnant magnetic field. It was observed that when positive external magnetic field (North-pole of a bar magnet) was applied to the sensor, the amplitude of the pick-up coil output signal was increased and the values of magnetic field signature, S1 was higher than S2.

The values of the positive and negative peaks were also different. The values of the positive peaks in S1 and S2 were $550 \mathrm{mV}$ and 330 $\mathrm{mV}$, respectively, while the value of the negative peaks in both $\mathrm{S} 1$ and S2 was $275 \mathrm{mV}$ (Figure 11a). When negative external magnetic field (South-pole of a bar magnet) was applied to the sensor (Figure 11b), the amplitude of the pick-up coil output signal was also increased, but in the opposite direction and the values of magnetic field signature, S1 was smaller than S2 (Figure 11b). The values of the positive and negative peaks were different too. The value of the positive peaks in both S1 and S2 was $275 \mathrm{mV}$ (Figure 11a), while the values of negative peaks in S1 and S2 were $330 \mathrm{mV}$ and $550 \mathrm{mV}$, respectively. These observations could be due to the simultaneous decreased permeability of the hysteresis loop in both reverse and forward magnetization curves.

\section{Conclusion}

In this research activity, a miniature fluxgate magnetometer with magnetic ring core in a square cross section of $2 \mathrm{~mm}$ was realized. The sensor was wire-wound based on traditional technology process. The 
Citation: Sadiq UA, Sadka AH, Oluyombo OW (2018) Optimization Design and Development of Sensing Coil and Analog Signal Conditioning Electronics for Fluxgate Magnetometer Sensor. J Biosens Bioelectron 9: 261. doi: 10.4172/2155-6210.1000261

Page 6 of 6

signal conditioning of the developed modified FOA fluxgate sensor was done by external electronics circuits. A square wave excitation current produced by a frequency oscillator circuit was fed to the excitation coil. The Helmholtz coils produced the external magnetic field to be measured. The second harmonic frequency of the induced voltage across the pick-up coil was measured with detection electronic circuit synchronized with the reference frequency from the square wave oscillator.

The introduction of the combined Firefly Optimization Algorithm (FOA) and the systematic optimization approach to FMS design problem in this research, by simultaneously finding the optimum dimensions and geometry of the sensor core, pick-up coil, and detection circuit, significantly improved the matching of the excitation and detection circuits. The developed optimized sensor for earth's magnetic field exploration showed good sensitivity of $97.09 \mathrm{mV} / \mu \mathrm{T}$ and linearity in the range of about $\pm 49.44 \mu \mathrm{T}$. The power consumption of the sensor was $131 \mathrm{~mW}$, the sensor electronics consumed $315 \mathrm{~mW}$, while the whole sensor system consumed $446 \mathrm{~mW}$. Hence, good sensitivity and the possibility of detecting magnetic field along two perpendicular directions make the developed FOA-based sensor suitable for portable compass application.

\section{References}

1. Lu CC, Huang J (2015) A 3-Axis miniature magnetic sensor based on a planar fluxgate magnetometer with an orthogonal fluxguide. Sensors 15: 1472714744.

2. Lv H, Liu S (2013) Research on MEMS technology of micro fluxgate sensor. Int J Digit Content Technol Appl 7: 1159-1167.

3. Frydrych P, Szewczyk R, Salach J (2014) Magnetic fluxgate sensor characteristics modeling using extended Preisach model. Acta Physica Polonica Series 126: 18-19.

4. Can H, Topal $\cup$ (2015) Design of ring core fluxgate magnetometer as attitude control sensor for low and high orbit satellites. J Superconductivity and Novel Magnetism 28: 1093-1096.

5. Indrasari W, Djamal M, Srigutomo W (2012) A magnetic distance sensor with high sensitivity based on double secondary coil of fluxgate. IOSR J Appl Phys 2: 29-35.

6. Kim YH, Kim Y, Yang CS, Shin KH (2013) Optimization of operation frequency of orthogonal fluxgate sensor fabricated with Co based amorphous wire. $J$ Magn 18: 159-162.

7. Lv H, Liu S (2014) Design and fabrication of low power consumption micro fluxgate sensor. Sensors and Transducers 182: 22-27.

8. Grosz A, Paperno E (2012) Analytical optimization of low frequency search coil magnetometers. IEEE Sensors J 12: 2719-2723.

9. Chen C, Liu F, Lin J, Wang Y (2015) Investigation and optimization of the performance of an air-coil sensor with a differential structure suited to helicopter TEM exploration. Sensors 25: 23325-23340.

10. Grosz A, Paperno E, Amrusi S, Zadov B (2011) A three-axial search coil magnetometer optimized for small size, low power, and low frequencies. IEEE Sensors J 11: 1088-1094.

11. Baschirotto A, Dallago E, Ferri M, Malcovati P, Rossini A, et al. (2010) A 2D micro-fluxgate earth magnetic field measurement system with fully automated acquisition setup. Measurement 43: 46-53.

12. Velasco QG, Román LM, Conesa RA, Jeréz F (2011) Design of a lowconsumption fluxgate transducer for high-current measurement applications. IEEE Sensors J 11: 280-287.

13. Zorlu O, Kejik, P, Teppan W (2010) A closed core micro-fluxgate sensor with cascaded planar FeNi rings. Sensors and Actuators A 162: 241-247.

14. Waheed OT, Rehman A (2011) Design and development of a fluxgate magnetometer for small satellites in low earth orbit. J Sp Tech 1: 78-82.

15. Ripka P (2001) Magnetic sensors and magnetometers. Artech House, Boston, MA

16. Cui ZJ (2013) Design of a novel excitation circuit for low perming error fluxgate. Advanced Materials Research 748: 859-863.

17. Miles DM, Bennest JR, Mann IR, Millling DK (2013) A radiation hardened digital fluxgate magnetometer for space applications. Geoscience Instrumentation Methodology Data System 2: 213-224.

18. Tumanski S (2013) Modern magnetic field sensors: A Review. Przegląd Elektrotechniczny 89: 1-12.

19. Yang XS (2013) Multi-objectives firefly algorithm for continuous optimization. Engineering Computation 29: 175-184.

20. Fairchild Semiconductor (2002) CD4049 Hex Inverting Buffer Manual. Fairchild Semiconductor Corporation.

21. Han F, Harada S, Sasada I (2012) Fluxgate and Search Coil Hybrid: A LowNoise Wide-Band Magnetometer. IEEE Transactions on Magnetics 48: 37003703. 